\title{
TATB/氟聚物PBX沿不同晶面力学性能的 分子动力学模拟
}

\author{
胡应杰 ${ }^{(1)}$ 黄玉成 ${ }^{(1)}$ 肖继军 ${ }^{(1)}$ 肖鹤鸣 ${ }^{(* *}$
}

(1) 南京理工大学化学系, 南京 210094; (2) 南京晓庄学院化学系, 南京 210017)

\begin{abstract}
摘要 用分子动力学(MD)方法, 模拟计算了著名钝感炸药 $\operatorname{TATB}(1,3,5$-三氨基-2, 4, 6-三硝基 苯)与四种氟聚合物 [ 聚偏二氟乙烯 $(\mathrm{PVDF}) 、$ 聚三氟氯乙烯 $(\mathrm{PCTFE}) 、$ 氟橡胶 $\left(\mathrm{F}_{2311}\right) 、$ 氟树脂 $\left.\left(\mathrm{F}_{2314}\right)\right]$ 构成的高聚物粘结炸药(PBX)的力学性能. 结果表明, 在 TATB 中添加少量氟聚物能有效改善其 力学性能; 沿 TATB 不同晶面与氟聚物“粘结”, 构成 PBX 的力学性能有所不同, 改善力学性能的 整体效应为 $(010) \approx(100)>(001)$.
\end{abstract}

\section{关键词 TATB 高聚物粘结炸药 力学性能 分子动力学}

TATB(1,3,5-triamino-2,4,6-trinitrobenzene)是著名 的高能钝感炸药. 以其为主体( $90 \%$ 以上)的高聚物粘 结炸药(PBX), 含少量(5\% 10\%)高分子粘结剂, 是军 民两用具有优异安全和力学性能的复合材料. 虽然 以实验测定 $\frac{114]}{1}$ 和以量子力学 $(\mathrm{QM})$ 、分子力学 $(\mathrm{MM})$ 、 分子动力学 (MD) 模拟计算 ${ }^{[59]}$ 炸药或高聚物的论著 已较多, 但迄今鲜见模拟PBX力学性能的任何报道, 且对此复杂体系的复杂受力情况, 其应力应变一般 关系也未见以实验方法加以测量. 因此，模拟PBX的 力学性能不仅具有重要理论意义, 符合化学学科从 “分子化学”向“材料化学”发展的大趋势; 而且由于实 际使用的不是单体炸药(化合物)而是混合炸药, PBX 即典型的混合炸药, 其配方设计迫切需要理论指导,
所以模拟其最重要的性能一一力学性能，也具有重 要的实用价值. 本文选择四种典型含氟聚合物 $\operatorname{PVDF}($ 聚偏二氟乙烯)、PCTFE(聚三氟氯乙烯)、 $F_{2311}$ (氟橡胶)和 $F_{2314}$ (氟树脂)与 $T A T B$ 构成双组分 PBX. 其中 $F_{2311}$ 和 $F_{2314}$ 分别是偏二氟乙烯与三氟氯乙烯按 照 $1: 1$ 和 $1: 4$ 摩尔数比共聚产生, 它们的分子式分 别为 $\mathrm{C}_{40} \mathrm{H}_{20} \mathrm{~F}_{51} \mathrm{Cl}_{11}$ 和 $\mathrm{C}_{40} \mathrm{H}_{8} \mathrm{~F}_{57} \mathrm{Cl}_{17}$, 端基视情况分别以 $\mathrm{H}$ 或F加以饱和. 通过先进的COMPASS ${ }^{[10]}$ 力场 MD周 期性计算, 求得纯TATB和PBX沿不同晶面的力学性 能(弹性系数、模量和泊松比), 发现添加少量粘结剂 即能有效改善炸药的力学性能, 还比较分析了不同 粘结剂及其沿TATB不同晶面作用对力学性能所产生 的不同影响，以期对PBX配方实验具一定指导作用. 


\section{1 模型构建与模拟方法}

\section{1 用自编程序处理 TATB 晶面}

$\mathrm{X}$ 衍射晶体结构表明 [11], TATB分子具平面构型, 分子内和分子间氢键使整个体系连成一片，使晶体 具有类似于石墨的二维层状结构. 为细致地考察高 聚物沿TATB不同晶面对其力学性能所产生的影响, 本文采用切割分面的模型加以研究.

以Materials Studio(MS)3.0.1年]软件包中切割分 面的方法, 将TATB沿(001)、(010)和(100)三个晶面方 向切割, 并分别置于具周期性边界条件的三个周期 箱中，每个周期箱在 $Z$ 轴(C方向)留有 $1 \mathrm{~nm}$ 的真空层. 各体系均含 90 个ТАTB分子, 共 2160 个原子. 为区别 TATB内层和表面结构的差异，体现TATB晶体表面的 特点，对TATB表面作“升温回火”处理. 以(001)面为 例，固定其内部四层原子和次外层上下各两层苯环 碳骨架结构, 令最外层原子不受任何限制. 先从初始 温度 $298 \mathrm{~K}$ 每 $20 \mathrm{~K}$ 升温一次, 至最高温度 $658 \mathrm{~K}$ 后每 $20 \mathrm{~K}$ 降温一次, 直至回到室温, 沿整个过程还要逐次 进行 M D 模拟。原 D i s c o v e r 模块中需要人
为地重复升温、降温和 MD 模拟等一系列繁琐操作. 我们以 btcl 语言自编程序，使“升温回火”过程一次性 交付微机处理，减少了处理此类问题的时间和难度. 处理后置于各周期箱中的 TATB 各晶面如图 1 所示. 一些计算细节见表 1 .

从图 1 可见, 与晶体内层的理想规则结构相比, 处理后的表面显得较为凌乱，该表面较为符合实际， 利于真实地反映氟聚物在其上的相互作用.

\section{$1.2 \mathrm{PBX}$ 建模和 MD 模拟}

以程序搭建链节均为 20 的四种氟聚物链, 经 Amorphous Cell模块处理并进行 2.5ns的MD模拟以获 得其平衡构象, 各取两根平衡链分别置于如图 1 所示 不同晶面上，即得氟聚物链在不同晶面上共 12 种 PBX的初始构型. 解除各PBX中对所有原子的束缚, 经MM优化后, 再在Z方向经适当压缩使其密度较接 近理论值. 压缩后的PBX视为正则系综(NVT), 充分 平衡后，视为等温等压系综 $(\mathrm{NPT})^{[13]}$. 模拟中力场的 选择及非键力的计算均同表 1 . 各分子起始速度按

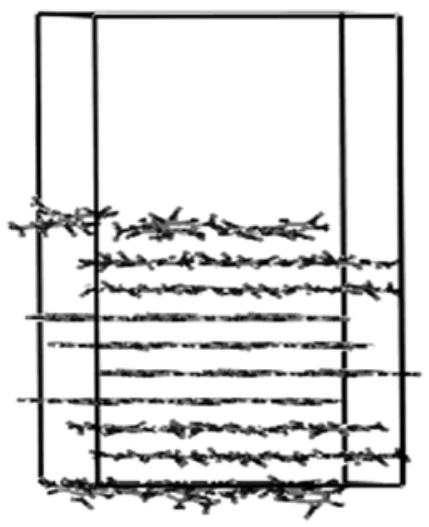

(001)

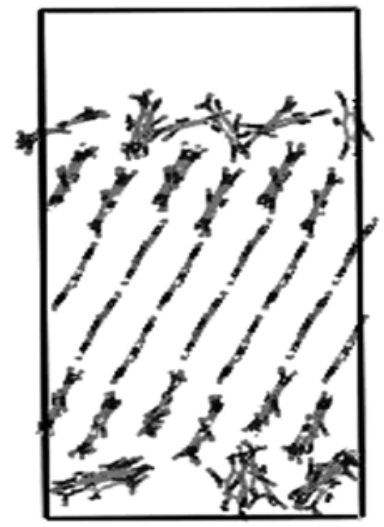

(010)

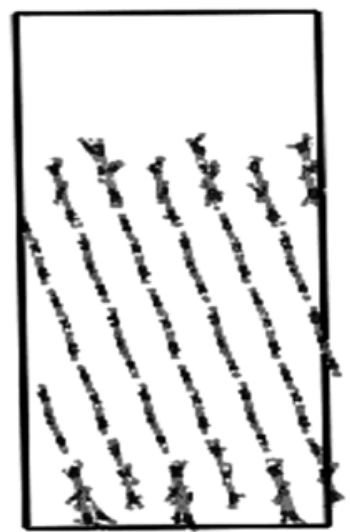

(100)

图 1 经处理的 TATB (001)、(010)和(100)面

表 1 升温回火计算细节

\begin{tabular}{ccccc}
\hline 力场 & 非键作用 $^{\text {a) }}$ & 加和方法 & 系综 & 初始温度 \\
\hline Compass & vdW, ${ }^{[10]}$ & Atom Based, Ewald & NVT & $298 \mathrm{~K}$ \\
最高温度 $^{658 \mathrm{~K}}$ & 温度变化幅度 & 回火次数 & 步长 & $1 \mathrm{fs}$ \\
\hline
\end{tabular}

a) 非键作用包括范德华和库仑作用, “Atom based”和“Ewald”方法分别用于计算范德华作用和库仑作用 
Maxwell分布取样，牛顿运动方程的求解建立在周期 性边界条件、时间平均等效于系综平均等基本假设之 上, 采用Velocity Verlet跳蛙法进行求解. 模拟过程中 位能截断采用球形截断法, 截断半径为 $0.95 \mathrm{~nm}$, 截断 距离之外的分子间相互作用能按平均密度近似方法 进行校正. MD模拟分两步: 首先在NVT系综中模拟, 使系统达到平衡; 然后转到NPT系综, 以统计各种宏 观量. 整个过程模拟温度选取 298K, 采用 Andersen ${ }^{[14]}$ 控温方法. 模拟压力为常压, 采用 Parrinello ${ }^{[15]}$ 控压方法. 时间步长设为 $1 \mathrm{fs}$, 模拟的总 步数为 50 万步, 前 30 万步用于体系平衡, 后 20 万步 用于统计分析, 最终模拟所得的 PBX密度约在 $1.82\left(\mathrm{~g} \cdot \mathrm{cm}^{-3}\right)$ 左右, 所取结果是其中五帧轨迹力学性 能计算的平均. 计算采用MS 3.0.1 软件包中自带的静 态分析方法.

\section{2 结果与讨论}

\subsection{PBX 体系的平衡}

体系平衡必须同时达到温度和能量的平衡. 以 $\mathrm{F}_{2314}$ 在 TATB (001)面上的 MD 模拟为例, 图 2 示出其 温度平衡曲线. 从该图可见，温度上下波动仅为 \pm $20 \mathrm{~K}$ 左右, 表明确已达到温度平衡. 其他 PBX 经 30 万步 $\mathrm{MD}$ 模拟也均达到温度平衡. 从平衡过程中所存 200 帧轨迹看, 各体系能量波动亦渐趋平缓. 图 3 示 出各氟聚物在 TATB (010)面后 100 帧轨迹的能量波 动, 由该图可见, 各帧轨迹能量偏差约为 $0.1 \%$ $0.5 \%$ 左右, 表明四种 PBX 体系已达能量平衡. 体系平衡 后经 200ps MD 模拟，每 1ps 收集一次体系内各原子 的运动轨迹, 保存全轨迹, 共收集 200 帧轨迹文件. 作为示例, 图 4 给出 $\mathrm{F}_{2314}$ 粘结 TATB 在三个面上的 MD 模拟结构.

\subsection{PBX 的力学性能}

基于 $M D$ 模拟所得体系的平衡运动轨迹，基于静 力方法分析原理, MS程序对体系实行多次单轴拉伸 与纯剪切形变操作后, 在原子水平上由维利公式求 得内应力张量, 于是弹性系数矩阵由数值法求相应 应力与应变的一阶偏导数而得到 ${ }^{[16]}$. 拉伸(或杨氏)模 量(拉伸应力与拉伸应变之比)和泊松比(横向应变与
纵向应变之比的负值)通过最小二乘法拟合求得, 进 而可求得其它有效各向同性力学性能, 如剪切模量、 体积模量和拉梅系数.

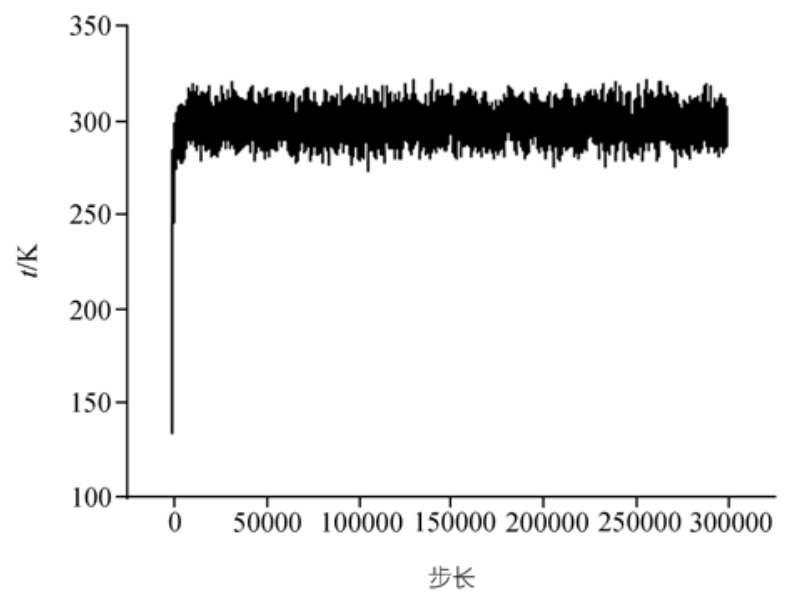

图 $2 \mathrm{~F}_{2314}$ 在平行 $\mathrm{TATB}(010)$ 面的 $\mathrm{PBX}$ 温度平衡曲线

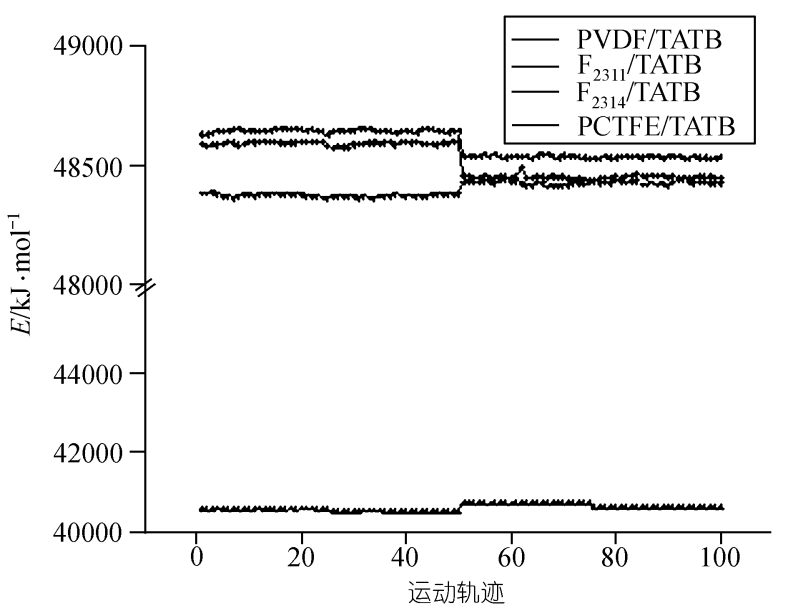

图 3 氟聚物在平行 TATB (010)面的 PBX 能量波动曲线

广义虎克定律是材料应力应变的最一般关系, 即应力用应变的线性组合表示, 组合系数 $C_{i j}$ 是 $6 \times 6$ 弹性系数矩阵元. 原则上讲, 材料的所有力学性质均 可从它的弹性系数矩阵导出. 由于弹性应变能的存 在, 即使极端各向异性体也满足 $C_{i j}=C_{j i}$, 即独立的弹 性常数只有 21 个. 物体对称性的提高可进一步减小 独立弹性系数的个数. 对于各向同性体, 仅有 2 个独 立的弹性系数, 故用两个拉梅系数(Lame coefficient) 


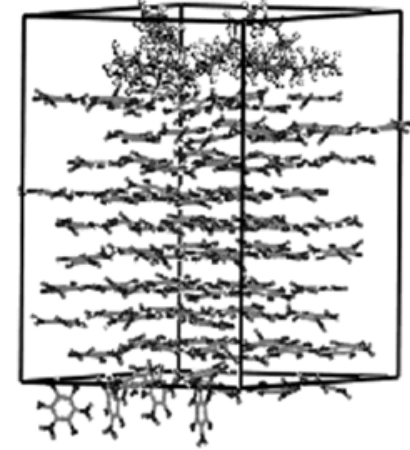

(001)

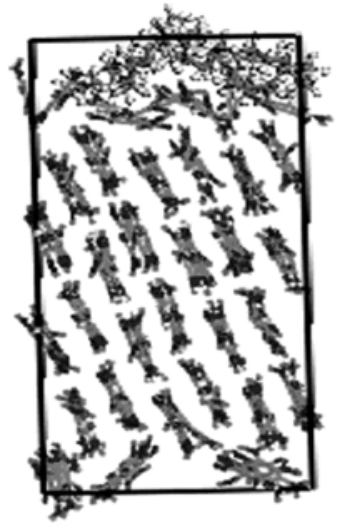

(010)

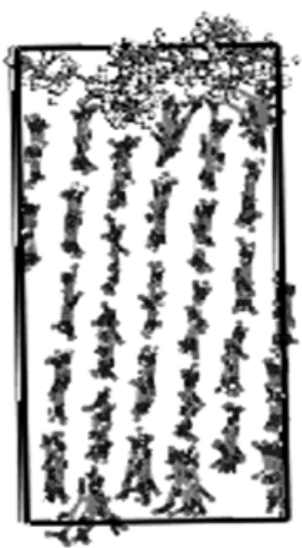

(100)

图 $4 \mathrm{~F}_{2314}$ 在 TATB 不同晶面上 MD 模拟结构

表 2 纯 TATB 及系列 PBX 在不同晶面上的弹性系数(GPa)

\begin{tabular}{ccccccccccccccc}
\hline & & $C_{11}$ & $C_{22}$ & $C_{33}$ & $C_{44}$ & $C_{55}$ & $C_{66}$ & $C_{12}$ & $C_{13}$ & $C_{23}$ & $C_{15}$ & $C_{25}$ & $C_{35}$ & $C_{46}$ \\
\hline & 纯 TATB & 44.9 & 38.4 & 16.9 & 15.9 & 0.1 & 0.9 & 10.8 & 1.9 & 5.5 & 0.1 & 0.2 & -0.5 & -0.7 \\
& PVDF & 8.1 & 16.9 & 10.8 & 1.4 & 2.4 & 3.5 & 4.5 & 5.3 & 3.7 & 0.5 & 0.6 & -1.3 & -1.0 \\
PBX & $F_{2311}$ & 8.4 & 14.7 & 11.8 & 1.5 & 1.0 & 3.0 & 3.7 & 4.3 & 3.7 & 0.9 & 0.6 & -0.9 & -0.4 \\
$(010)$ & $F_{2314}$ & 8.5 & 16.3 & 9.8 & 1.9 & 2.0 & 3.6 & 4.3 & 5.0 & 3.3 & 0.0 & 0.1 & -1.5 & -0.7 \\
& PCTFE & 7.8 & 14.7 & 9.0 & 1.3 & 0.9 & 2.5 & 3.0 & 3.4 & 2.4 & 0.2 & -0.2 & -0.2 & -0.1 \\
& PVDF & 20.8 & 9.4 & 12.2 & 0.9 & 2.2 & 0.6 & 4.5 & 3.1 & 3.2 & -0.3 & 0.0 & 0.2 & 0.6 \\
PBX & $F_{2311}$ & 19.0 & 7.6 & 8.7 & 1.2 & 3.1 & 1.1 & 2.0 & 2.5 & 2.0 & -0.6 & -0.7 & -0.4 & -0.1 \\
$(100)$ & $F_{2314}$ & 17.3 & 7.7 & 9.4 & 0.9 & 2.8 & 1.4 & 3.4 & 2.7 & 3.8 & -0.2 & 0.3 & 0.2 & 0.0 \\
& PCTFE & 19.3 & 7.8 & 6.8 & 0.9 & 2.3 & 1.0 & 2.8 & 3.0 & 3.7 & 0.6 & 0.1 & -0.9 & 0.0 \\
& PVDF & 27.9 & 22.7 & 7.5 & 8.9 & 0.7 & 0.8 & 4.4 & 1.6 & 1.7 & 0.1 & 0.1 & 0.1 & 0.0 \\
PBX & $F_{2311}$ & 24.4 & 24.8 & 6.4 & 9.6 & 0.8 & 0.8 & 6.0 & 1.2 & 1.6 & 0.1 & 0.2 & 0.0 & 0.0 \\
$(001)$ & $F_{2314}$ & 18.9 & 19.4 & 7.2 & 7.9 & 1.1 & 1.0 & 4.1 & 2.6 & 2.7 & 0.1 & 0.4 & 0.0 & 0.0 \\
& PCTFE & 20.2 & 20.1 & 6.0 & 8.4 & 0.9 & 0.9 & 3.7 & 2.4 & 2.8 & -0.1 & 0.1 & -0.1 & -0.2 \\
\hline
\end{tabular}

即可求出各模量和泊松比.

考察纯TATB和PBX的弹性系数矩阵, 由表 2 可 见, 除对角元 $C_{i i}$ 和 $C_{12} 、 C_{13} 、 C_{23}$ 共 9 个矩阵元外, 其 余各值很小, 多数接近为 0 , 可见所模拟的TATB和系 列PBX近似为正交各向异性弹性体 ${ }^{[17]}$. 对纯TATB而 言, 其 $C_{11} 、 C_{22}$ 很大, 说明产生同样的形变需承受很 大的应力; 而其他的系数较小, 表明 TATB晶体具有 明显的各向异性，与其具有石墨层状平面结构相一 致 [11]. 与纯TATB的 $C_{i j}$ 比较, 各PBX的 $C_{11} 、 C_{22} 、 C_{33}$ 和 $C_{44}$ 下降很大, $C_{13} 、 C_{31} 、 C_{55}$ 和 $C_{66}$ 却有所上升; $\mathrm{PBX}$ $C_{i j}$ 的平均化趋势, 反映添加粘结剂减小了体系的各 向异性，增加了各向同性.

表 3 示出TATB及其与PVDF、PCTFE、 $\mathrm{F}_{2311}$ 和 $\mathrm{F}_{2314}$
构成的PBX沿不同晶面的“有效各向同性力学性质” 一拉伸模量、体积模量、剪切模量和泊松比. 因无文 献值可类比, 故这里只将PBX与纯TATB的相应结果 进行比较. 由表 3 可见, 与纯TATB的(拉伸、体积和 剪切)模量相比, 所有 PBX的模量均系统地明显下降. 模量减小, 表明材料刚性减弱, 同时柔性塑性增强、 脆性降低. 因在我们模拟的PBX中, 高聚物含量只占 5\% 10\%, 与实际PBX中高聚物与基炸药之重量比相 符. 由此可见, 高聚物作为添加(粘结)剂少量混入炸 药, 确能有效地改善其力学性能. 这与新近报道的 HMX (奥克托金)基PBX力学性能的MD模拟结果相一 致 ${ }^{[18]}$.

若对各 $\mathrm{PBX}$ 的相同晶面进行比较, 则发现 
表 3 四种聚合物在 TATB 不同晶面上的力学性能(GPa)

\begin{tabular}{|c|c|c|c|c|c|}
\hline & & 杨氏（拉伸）模量 & 体模量 & 剪切模量 & 泊松比 \\
\hline \multicolumn{2}{|c|}{ 纯 TATB } & 31.5 & 15.2 & 13.7 & 0.15 \\
\hline \multirow{4}{*}{ PBX (010) } & PVDF & 9.5 & 3.7 & 7.0 & 0.27 \\
\hline & $\mathrm{F}_{2311}$ & 9.7 & 3.9 & 6.5 & 0.25 \\
\hline & $\mathrm{F}_{2314}$ & 9.4 & 3.7 & 6.6 & 0.26 \\
\hline & PCTFE & 9.2 & 3.8 & 5.5 & 0.22 \\
\hline \multirow{4}{*}{ PBX (100) } & PVDF & 12.8 & 5.4 & 7.0 & 0.19 \\
\hline & $\mathrm{F}_{2311}$ & 10.9 & 4.6 & 5.6 & 0.17 \\
\hline & $\mathrm{F}_{2314}$ & 10.0 & 4.1 & 6.0 & 0.22 \\
\hline & PCTFE & 10.0 & 4.1 & 5.8 & 0.21 \\
\hline \multirow{4}{*}{ PBX (001) } & PVDF & 18.8 & 8.4 & 8.2 & 0.12 \\
\hline & $\mathrm{F}_{2311}$ & 17.7 & 7.8 & 8.1 & 0.13 \\
\hline & $\mathrm{F}_{2314}$ & 14.1 & 6.0 & 7.0 & 0.17 \\
\hline & PCTFE & 14.4 & 6.2 & 7.1 & 0.16 \\
\hline
\end{tabular}

PCTFE对TATB力学性能的改善略优于其它氟聚物. 前文 [19]关于PBX结合能的MD模拟结果表明，四种氟 聚物与 TATB 结合能的排序为 $\mathrm{PVDF}>\mathrm{F}_{2311}>$ $\mathrm{F}_{2314}>\mathrm{PCTFE}$, 以 PVDF与TATB的界面氢键作用较强. 是否界面结合能大会导致其脆性增大、柔性减小呢? 这是有可能的. 因为柔性或塑性主要源自高聚物的 内旋转, 而界面分子间作用对内旋转起阻碍作用. 当 然这有待进一步深入研究. 但综合前文 ${ }^{[19]}$ 和本文可 见, 优选实用粘结剂, 需要综合考虑结合能和力学性 能两个方面.

细致比较各 PBX 不同晶面的计算结果, 发现沿 三个晶面添加粘结剂对改变 TATB力学性能的效应明 显不同，其改善效应的排序为： $(010) \approx(100)>(001)$. 由于 TATB 分子间和分子内的氢键较强, 同层(与 (001)面平行)分子间作用很强，而分子层间距离为 $0.33 \mathrm{~nm}$, 是 van der Walls 半径的两倍, 即层与层之间 作用力很弱. 因此, 沿(001)面添加氟聚物对 TATB 力 学性能的改善不如沿其它面作用强, 对(010)和(100)面 施力较之对(001)面施力, 其应变效应自然显著得多.

比较泊松比的计算结果亦可见，沿(001)面添加 粘结剂, 其值与纯 TATB 的泊松比相差很小, 均在 0.15 左右, 而其余面的相应结果均明显增大 $(0.2 \sim$ $0.3)$. 通常塑料的泊松比为 $0.2 \sim 0.4$, 可见 PBX 已具 塑料的某些性质.

\section{3 结论}

通过对纯 TATB 和 TATB/氟聚物 PBX 的力学性

能的 MD 模拟研究，导致如下结论:

(1) 首次报道了 TATB 基系列 PBX 沿不同晶面的 弹性系数、模量和泊松比;

(2) 在 TATB 中添加少量氟聚物能有效改善其力 学性能;

（3）沿 TATB 三个晶面与氟聚物粘结, 对 TATB 力学性能的改善效应为 $(010) \approx(100)>(001)$;

(4) 系列高聚物对 TATB力学性能的改善效应及 其与 TATB的结合能 ${ }^{[19]}$ 并不存在平行递变关系，实际 优选粘结剂必须综合考虑结合能和力学性能两个方 面.

\section{参 考 文 献}

2 ing agents for TATB/HTPB polymer bonded explosives. Philos Trans R Lond A, 1992, 339: 321 333

3 宋华杰, 董海山, 郝 芗. TATB、HMX 与氟聚合物的表面能研 究. 含能材料, 2000, 8: 104 107

4 董海山, 周芬芬. 高能炸药及相关物性能. 北京: 科学出版社, 1989. $1 \sim 45$

5 Xiao Heming, Li Jinshan, Dong Haishan. A quantum-chemical study of PBX: intermolecular interactions of TATB with $\mathrm{CH}_{2} \mathrm{~F}_{2}$ and with linear fluorine-containing polymers, Journal of Physical Organic Chemistry, 2001, 14: 644 649 [DOI]

6 李金山, 肖鹤鸣, 董海山. PBX 的量子化学研究-TATB 与甲 烷、聚乙烯的分子间相互作用. 爆炸与冲击, 2000, 20(3): $221 \sim 227$

7 肖鹤鸣, 居学海. 高能体系中的分子间相互作用. 北京: 科学 出版社, 2003 
8 Thomas D S, Ralph M, Dmitry B, et al. A molecule dynamics simulation study of elastic properties of HMX. J Chem Phys, 2003, 119(14): 7417 7426 [DOI]

9 杨小震. 分子模拟与高分子材料. 北京: 科学出版社, 2002

10 Sun $\mathrm{H}$. The parameterization and validation of a condensed-phase optimized ab initio forcefield. J Chem Phys, 1998, B102: 7338 7364

11 Cady H H, Larson A C. The crystal structure of 1,3,5-triamino2,4,6-trinitrobenzne. Acta Cryst, 1965, 18(3): 485 496

12 Materials Studio 3.0,Accelys: San Diego, Ca.2004

13 Heermann D W. 秦克成译.理论物理学中的计算机模拟方法. 北 京: 北京大学出版社, 1996

14 Andersen H C. Molecular dynamics simulations at constant pressure and/or temperature. J Chem Phys, 1980, 72: 2374 2383
[DOI]

15 Parrinello M, Rahman A. Strain Fluctuations and Elastic Constants. J Chem Phys, 1982, 76: 2662 2666 [DOI]

16 Weiner J H. Statistical Mechanics of Elasticity. New York: John Wiley, 1983

17 徐芝纶. 弹性力学. 北京: 高等教育出版社, 1990

18 Xiao J J, Fang G Y, Ji G F ,et al. Simulation investigations in the binding energy and mechanical properties of HMX-based polymer-bonded explosives. Chinese Science Bulletin, 2005, 50(1): $21 \sim 26$

19 黄玉成, 胡应杰, 肖继军, 等. TATB 基 PBX 结合能的分子动力 学模拟. 物理化学学报, 2005, 21(4): 425 429 